\title{
ASOCIACIONISMO JUVENIL
}

\section{Rosa Maㅗ Sandín Romano}

RESUMEN. El presente artículo muestra el análisis y las primeras conclusiones de un estudio sociológico realizado en La Rioja, sobre el nivel de Asociacionismo Juvenil. La investigación ha sido subvencionada por la Consejería de Juventud del Gobierno de La Rioja.

Las conclusiones obtenidas nos permiten conocer más la demanda en materia de asociacionismo juvenil así como, la necesidad de transformar las iniciativas existentes o crear otras nuevas.

ABSTRACT. This article shows the analysis and the first clonclusions about a sociologic investigation realizated in La Rioja, about the level of young associationism. This investigation has been greated by the youngs Advicer Institution from the Government of La Rioja.

The conclusions obtained allow us to know more about the demand of young associationism and both the necessities to change the actually initiatives or to creaty new others.

\section{Introducción}

Existen en la sociedad riojana muchas asociaciones juveniles constituidas como tal; hay además grupos juveniles reconocidos como pueden ser parroquiales, colegiales, asociaciones de estudiantes, etc. donde los jóvenes interesados pueden introducirse y encontrar personas que comparten sus intereses e inquietudes, con los que poder realizar actividades que contribuyan a su desarrollo personal y en cierta manera, a la mejora de la convivencia social.

Sin embargo, preocupa según los datos que se barajan, que los jóvenes no tienen motivación para integrarse en estos grupos. La cuestión que alarma y objeto, en cierta medida, de esta investigación es intentar descubrir cuáles pueden ser algunos de los motivos. Se ha realizado para ello un estudio sociológico becado por la Consejería de Juventud del Gobierno de La Rioja, que nos permite introducirnos en el mundo juvenil, hablar con ellos y darles la oportunidad de expresar sus sentimientos reales sobre el tema.

Vamos a verificar en este estudio algunas de las hipótesis planteadas en un primer momento. Ello nos puede dar una idea de qué es lo que falla, y poder solucionarlo 
para facilitar y motivar que los jóvenes se asocien a instituciones o grupos que les aporten valores positivos en la difícil etapa en la que se encuentran e intentar evitar que cada vez más jóvenes beban sin control, fumen y se introduzcan en ambientes poco recomendables con unas normas de vida muy poco aconsejables y saludables.

\section{Objetivos}

En el momento de plantearse este estudio, nos centramos en unos objetivos fundamentales, que pasamos a explicar a continuación:

- Averiguar cuáles son algunos de los motivos por los que los jóvenes no se sienten motivados para ser parte integrante de una Asociación Juvenil;

- Explicar en la medida de lo posible los motivos que de ellos se hayan concluido;

- Encontrar posibles vías de solución a este problema de la sociedad actual;

- Acercarnos lo más posible a la realidad vivida por los jóvenes riojanos;

- Descubrir qué tipo de asociaciones resultan más interesantes para los jóvenes de estas edades;

- Averiguar qué actividades pueden interesar más para ayudar a los jóvenes a asociarse;

- Saber cómo podemos de alguna manera ayudar a las nuevas generaciones a abrirse al mundo de una forma integrada y organizada.

\section{Destinatarios - Metodología}

La investigación se ha dirigido hacia los jóvenes logroñeses, con edades comprendidas entre 17 y 21 años, y que estuvieran realizando estudios de COU o universitarios en la Universidad de La Rioja, de seis titulaciones diferentes: Químicas, Ingeniería, Empresariales, Derecho, Letras (Humanidades y Filologías), y Magisterio.

Para la realización de este estudio se han pasado unos cuestionarios a cada uno de los jóvenes referidos al asociacionismo. Aunque el número total de cuestionarios ha sido 800, mayor de lo que en un principio se pensó (700), hay que tener en cuenta que todos ellos no son válidos, ya que algunos no son aceptables por no corresponderse con las edades objeto del estudio. Otros han resultado nulos, ya que no han aportados datos imprescindibles para las estadísticas, como puede ser el sexo, o la edad. Por lo tanto, la cantidad final válida ha sido 596 cuestionarios (aunque en realidad son 680 contabilizados).

\begin{tabular}{|l|c|c|c|c|c|c|c|c|}
\hline IIIIIIIIII & COU C" & COU LE & INGENIE & QUIMICA & EMPRES & DERECH & MAGIST & HUMANI \\
\hline HOMBRE & $\mathbf{3 7}$ & 17 & 112 & $\mathbf{3 5}$ & $\mathbf{2 8}$ & 25 & 12 & 18 \\
\hline MUJER & $\mathbf{3 4}$ & 19 & 15 & 33 & 66 & 53 & 57 & 36 \\
\hline TOTALES & $\mathbf{7 1}$ & $\mathbf{3 6}$ & $\mathbf{1 2 7}$ & $\mathbf{6 8}$ & $\mathbf{9 4}$ & $\mathbf{7 8}$ & $\mathbf{6 9}$ & $\mathbf{5 4}$ \\
\hline
\end{tabular}


Como podemos ver el mayor número de encuestados corresponde a Ingeniería Técnica; además podemos comprobar cómo es la única titulación en la es marcada la diferencia entre sexos, continúa siendo una carrera de hombres, aunque estas tendencias se van modificando notablemente como vemos en el resto de titulaciones, y sobre todo en Química, que parece haber cambiado casi de dirección. En el resto se aprecia una marcada predominancia del sexo femenino.

Hemos dicho que hemos aumentado el número de cuestionarios de lo que en un principio pensábamos, dado el número de matriculados en las clases, que es variable en las diferentes titulaciones, y previendo que algunos podrían resultar no válidos, tal como ha sido el caso. Por ese mismo motivo de la variación entre matriculados a una clase (sobre todo en estudios universitarios) y los realmente asistentes, encontramos al hacer el recuento, que algunas carreras tiene mayor número de encuestados que otras. Sin embargo, en todas se han planteado de la misma manera y con el mismo interés.

Las clases que se han seleccionado han sido por número de alumnos, curso y según la disponibilidad del profesor y horario.

El estudio sociológico presente ha sido becado por la Consejería de Juventud en casi la totalidad de los gastos. Es posible que se realice una nueva investigación con el mismo objetivo, pero con diferentes destinatarios, de edades más tempranas, para determinar los posibles antecedentes a estos resultados.

Una vez realizado el recuento de encuestados y el vaciado de la información, nos hemos ayudado de diferentes técnicas informáticas para las conclusiones y la baremación final.

\section{Hipótesis Planteadas}

Veamos a continuación los resultados según las hipótesis planteadas en el proyecto. En un primer momento existe una Hipótesis General, que es aquella que nos da los resultados concluyentes del estudio, a ella se ha llegado a través de una serie de Hipótesis Secundarias planteadas para explicar y verificar la general, son causa de ésta.

\subsection{Hipótesis General}

"LOS JÓVENES RIOJANOS NO SE ASOCIAN"

\section{ASOCIADOS I NO ASOCIADOS}

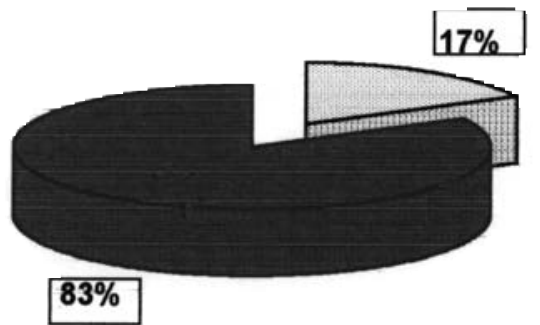

$\square$ ASOCIADOS

ENO ASOCIADOS

$83 \%$ 
Vemos pues en nuestra gráfica cómo la casi totalidad de los jóvenes encuestados no pertenece actualmente a ningún grupo juvenil, aunque algunos de ellos han pertenecido con anterioridad. Tan sólo el 17 \% sí pertenecen actualmente a alguna asociación juvenil; hemos incluido como tal a jóvenes que pertenecen al grupo juvenil de la parroquia que son la inmensa mayoría de los que ha contestado afirmativamente. Con lo cual si además limitamos los campos de actuación, el porcentaje sería mucho menor.

Por tanto, como hemos podido comprobar, la hipótesis general en este caso se verifica. Son cifras y datos estadísticos creo no sorprenden a nadie, ya que la realidad lo muestra todos los días.

Las asociaciones juveniles, se ven obligadas a realizar campañas de asociacionismo para mantenerlas en funcionamiento y continuar ayudando a jóvenes que se encuentran en situaciones socialmente difíciles. El Ayuntamiento junto con la Comunidad Autónoma y el Gobierno de La Rioja hacen lo propio con instituciones locales muy conocidas por todos. Sin embargo, los datos son bastante reveladores que hablan por sí solos. Existe una gran oferta pero muy escasa demanda; nuestra cuestión ahora debe ser dirigirnos hacia el equilibrio de las tendencias.

Pasemos a continuación a ver con más detalle las hipótesis planteadas en un principio y los resultados obtenidos, para que con ello nos ayuden a entender un poco más los motivos que llevan a esos datos tan alarmantes.

\subsection{Hipótesis Específicas}

\section{HIPÓTESIS 1}

\section{"LOS JÓVENES RIOJANOS ESTÁN POCO INFORMADOS DE LA REALIDAD DE LAS ASOCIACIONES JUVENILES"}

Según los datos obtenidos, hemos llegado a esta conclusión a través de varias preguntas de nuestro cuestionario. Los jóvenes no están realmente informados del funcionamiento de las asociaciones juveniles, o bien la información que tienen es escasa, con lo cual la conclusión que sacan de las mismas es errónea. Es curioso como algunos de los que no están asociados, sí que han pertenecido a algún grupo juvenil alguna vez, pero a la hora de responder sobre el funcionamiento, confiesan que no conocen realmente cómo es y cómo funciona una asociación juvenil; también hay que añadir que el interés a priori que tienen por descubrirlo es escaso. Veamos a continuación con mayor detenimiento la gráfica que nos muestra los resultados numéricos.

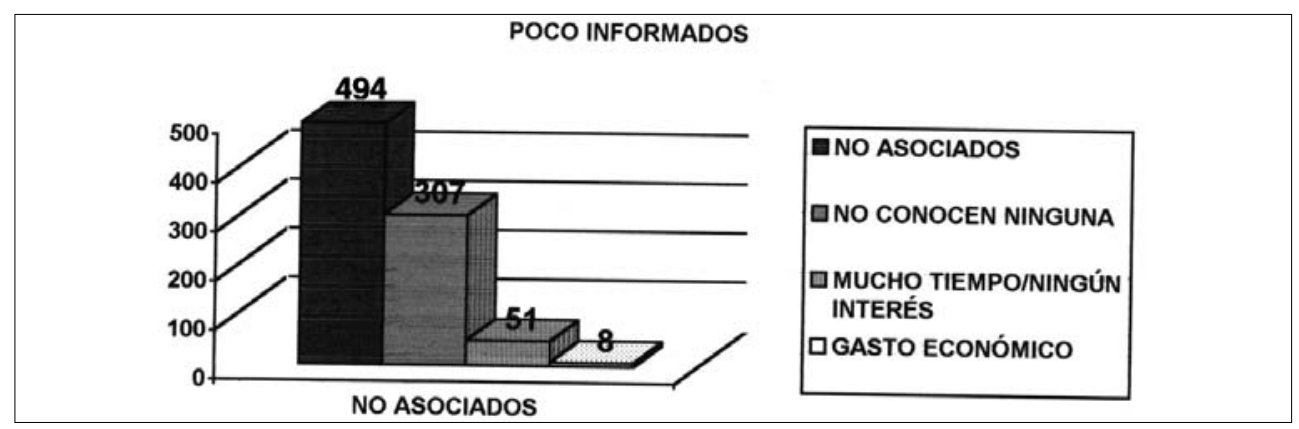


Como podemos observar, de los 494 jóvenes asociados, hay muy pocos que reconozcan conocer el funcionamiento de una asociación, al menos lo suficiente. Tan sólo 51 creen además que el pertenecer a alguna asociación juvenil les ocuparía demasiado tiempo, lo cual ya para ellos, en principio no parece ser un factor determinante para no asociarse. Y es significativo cómo tan sólo un grupo muy escaso, cree además que el pertenecer a un grupo juvenil supondría un gasto económico por su parte; parece por ello que el gasto o bien no se lo habían planteado, o no les preocupa, o realmente saben que no supone tal gasto en la mayoría de ellas, ya que el resto, 486, no lo consideran importante como para reflejarlo en las preguntas planteadas. Lo que nos llevará a cuestionarnos si existen ideas erróneas quizá por nuestra información en campañas y actividades realizadas.

Existe más de un 35 \% que han pertenecido alguna vez a un grupo de estas características y sin embargo en estos momentos, pocos años después de integrarse han decidido salir de la asociación. Lo que debe preocuparnos son los motivos que les han llevado a ello para intentar darle solución en el centro de la realidad. Este es otro de los objetivos que nos hemos planteado en este estudio y que a lo largo del mismo intentaremos darle respuesta.

Quizá estos datos nos ayuden a analizar las campañas de promoción que se han estado realizando, ver dónde pueden estar lo errores para que la información no llegue correcta o simplemente es que no llega.

Sin embargo, si las condiciones cambian parece todo indicar que a los jóvenes no les importaría integrarse en grupos de diversos tipos; a partir de aquí, como también veremos, estudiaremos cuáles son los más significativos para ellos.

\section{HIPÓTESIS 2}

\section{"LOS JÓVENES RIOJANOS NO ENCUENTRAN EN LAS ASOCIACIONES NADA QUE LES PUEDA INTERESAR"}

Como vamos a ver, tal como hemos comentado, los jóvenes encuestados creen que las asociaciones juveniles o los grupos juveniles, no les van a aportar nada que les pueda interesar a nivel personal, social o cultural, con lo cual disminuye el interés por asociarse, ya que en definitiva, no le encuentran sentido.

¿APORTAN ALGO QUE INTERESE?

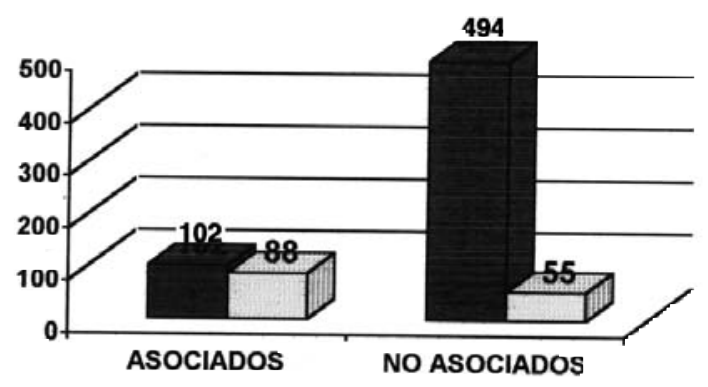

ASOCIADOS/NO ASOCIADOS

DALGO/NADA

INTERESANTE 
De los 102 que está asociados, 88 opinan que el pertenecer a un asociación juvenil satisface sus expectativa tanto personales, como sociales y culturales. Dicho de otra manera, más del $86 \%$ piensan que es satisfactorio, están contentos y no se plantean abandonar sus actividades, les es útil. Tan sólo el restante casi $14 \%$, piensa que a pesar de pertenecer, no les sirve de mucho en ningún sentido. Es decir, que una vez que están integrados realmente en la asociación sí que encuentran motivo para ello.

En la misma línea, vemos que de los 494 jóvenes encuestados y que no pertenecen a ningún grupo, tan sólo 55 creen que no les interesa en ningún sentido pertenecer, ya que no les servirá de nada en absoluto, y por lo tanto les supone una pérdida de tiempo. O sea, que 439 creen que a lo mejor pertenecer a una asociación sí puede aportarles algo nuevo e interesante, aunque en un principio las expectativas que tiene de ello no son esperanzadoras; son un poco susceptibles, y ante eso prefieren no arriesgarse y permanecer sin estar en ningún grupo.

Es ante este casi 89 \% de la población no asociada donde debemos trabajar y centrarnos para ayudarles a conocer la realidad y solucionar las posibles dudas que se les planteen. Nuestro trabajo no consiste sólo en mantener los interesados sino en intentar que esos "dudosos" se decanten por una tendencia importante e interesante para el desarrollo de sus propias vidas y personalidades, además de contribuir con ello al desarrollo de la sociedad futura en general. Tengamos siempre presente que sólo es un $11 \%$ los que no tienen ningún interés y a los que nuestras campañas y dedicaciones no les va a llegar.

\section{HIPÓTESIS 3}

\section{"LOS JÓVENES RIOJANOS CREEN QUE PERTENECER A UNA ASOCIACIÓN JUVENIL LES OCUPARÍA DEMASIADO TIEMPO"}

Tal como vemos en el gráfico, los jóvenes piensan que sí les ocuparía demasiado tiempo el pertenecer, aunque no todos lo tienen tan claro, sólo el $50 \%$ se ratifica en esta idea dentro de los no asociados. El porcentaje disminuye si hablamos de los que sí pertenecen a algún grupo, el $10 \%$, prácticamente, tienen la misma idea. Queda por lo tanto confirmada la hipótesis planteada.

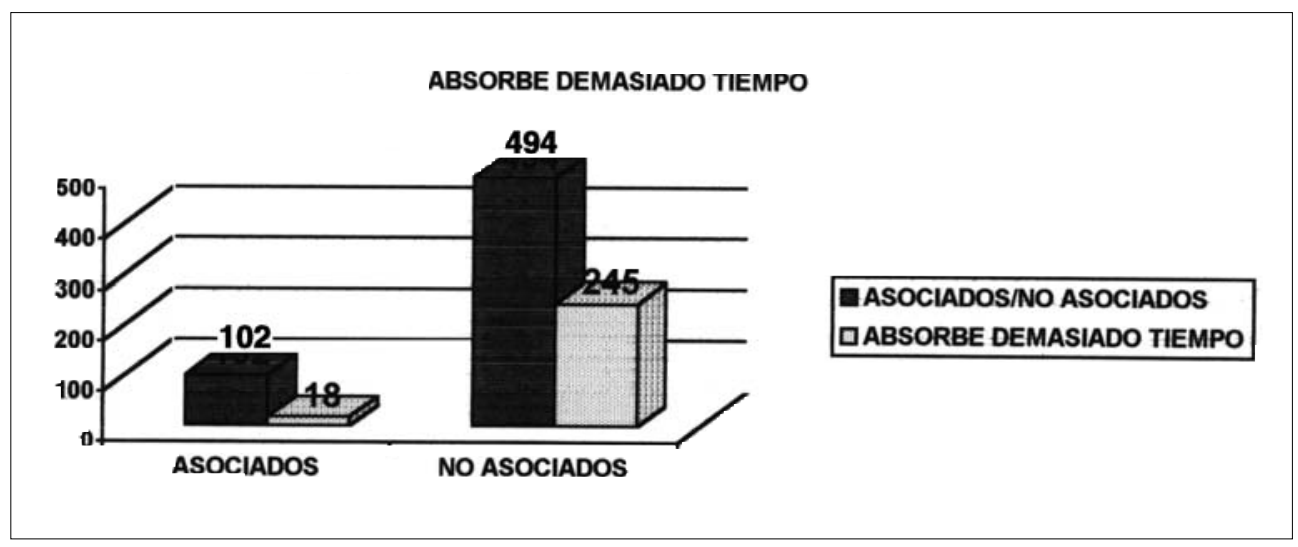


Con todo ello llegamos a que los jóvenes no tiene tan claro de que realmente la asociación les absorba demasiado tiempo y que no les dé nada que les interese; sin embargo continúan sin interés en la asociación, con lo cual deberíamos analizar cuál es el motivo real para que ellos no estén motivados. Con todos estos datos generales podemos ya sacar una conclusiones claras de este estudio, ya que todas las hipótesis han sido confirmadas.

Hagamos una recopilación de los datos de que disponemos. En principio vemos que la población no tiene datos suficientes por distintos motivos para conocer las asociaciones, están poco informados del funcionamiento real de las mismas, y eso a algunos les lleva a pensar que el estar asociado a alguna le supondría un desembolso económico. No tienen información suficiente ya que además piensan que le supondría demasiado tiempo, y eso sí que les preocupa, ya que lo consideran incompatible con sus actividades diarias, con sus estudios y deportes y es un factor suficientemente fuerte que determina su comportamiento frente al asociacionismo. $Y$ si además añadimos el concepto de que no van a encontrar en ellas nada que les pueda resultar interesante, en nivel de desinterés aumenta. Nos debería preocupar que el hecho de conocer gente nueva y de unirse por una sociedad mejor, por realizar actividades por y para los demás y por salir de una sociedad consumista y cómoda, aunque tecnológicamente avanzada, no es suficiente para que la población se plantee realizar un giro en su línea de actuación. Se siguen a pesar de todo pautas muy determinadas por la mayoría, por las corrientes y tendencias más avanzadas y modernas, por disfrutar antes de lo que debería ser más tarde. Y todo esto nos lleva a que los jóvenes no tienen interés en integrarse en grupos de iguales.

Con todo ello sí que deberíamos hacer ahora un estudio de nuestra lucha, nuestras campañas y nuestras intenciones para analizar si realmente tal y como lo hemos planteado se ha hecho pensando en ello, o pensando en lo que nosotros creíamos que les interesaba a ellos.

Para intentar aclarar más dudas, nuestra investigación sociológica va más allá recopilando nuevos datos de gran interés que nos facilitan ampliar las conclusiones.

\section{Conclusiones}

\section{RESUMEN DE ASOCIADOS}

Los datos aquí concluidos son bastante equilibrados e igualados, lo que nos demuestra que la opinión es bastante somera por parte de los que pertenecen a grupos juveniles. De hecho la práctica totalidad ha resumido su aportación en los informes en que aconsejarían a los demás a asociarse, el 97 \%. Eso puede darnos una idea de la situación que viven los jóvenes una vez que están integrados dentro de un grupo. Sus vivencias resultan positivas, tienen claro continuar en un 95 \% y poco mayor es el porcentaje para los que están convencidos de haber hecho buenas amistades dentro de las asociaciones, y eso sí que resulta para la población un factor motivante para la continuidad y el asociacionismo. Se contrapone a todos estos datos que muy pocos de ellos piensan de forma negativa, como pueda ser que les absorba demasiado tiempo, (tan sólo 18 de 102). Otro factor a tener en presente es la relación que hay entre pertenecer a una entidad de estas características y la lejanía o cercanía de la institu- 
ción del domicilio del joven; sin embargo no lo consideramos factor determinante ya que tan sólo el 65 \% se encuentra cerca de la asociación a la que pertenece (63 de los 102 asociados). Ello nos indica que una vez integrados los factores que antes podían resultar negativos sufren un giro de $180^{\circ}$ y se convierten en datos poco relevantes que no condicionan la continuidad o permanencia en al asociación.

\section{RESUMEN DE NO ASOCIADOS}

En este caso dentro de los que actualmente no pertenecen a ninguna asociación o grupo juvenil (494), hay una cuarta parte (162 jóvenes) que no les parece realmente interesante el pertenecer, no le encuentran aliciente y están totalmente desmotivados. Existe un $16 \%$ (73 de los encuestados que no son asociados), que creen que no sirve tampoco para el desarrollo personal, que en todo caso la diferencia de ambos datos que piensan que de algo te puede servir, aunque en principio no resulten atrayentes ni interesantes.

Sin embargo, es importante destacar que 325 de los 494, es decir, prácticamente el $66 \%$ de los encuestados que no pertenecen, tiene algún amigo que sí pertenece a una asociación o grupo juvenil. Además es significativo, que a pesar de que hemos visto que existían dudas en cuanto a la firmeza de los datos referentes al interés de asociarse, como comprobamos en las Hipótesis planteadas, 303 de los encuestados afirman que no tienen intenciones, al menos a corto plazo de asociarse. Son estos niveles los que nos deberían preocupar ya que es donde realmente podemos actuar.

Como veremos más adelante, un gran grupo de casi el $56 \%$ de la población no asociada creen que el pertenecer a él les supondría una incompatibilidad con sus estudios actuales, por las actividades desarrolladas en la asociación, por su horario o por el tiempo que hay que dedicarles. Este podría ser un dato bastante significativo de por qué los jóvenes no se asocian. Y tan sólo 194, de este apartado aseguran vivir cerca de una asociación; con lo cual el factor de cercanía o lejanía no parece determinante a la hora de tener intenciones de asociarse; además hemos de destacar que la diferencia de 300 encuestados no viven cerca de ninguna asociación o no lo creen, ya que si tenemos presente que la inmensa mayoría no las conocen, a lo mejor tampoco saben que una asociación que tiene cerca es tal. Y teniendo presente el número de asociaciones que hay creadas en Logroño, es difícil que alguien no viva cerca de alguna.

Como hemos podido comprobar, los resultados son diferentes a los de la población asociada, ya que ante la misma pregunta y situación, las interpretaciones son diferentes, tiene conciencia de ello en variada forma. Ellos no lleva a deducir que las interpretaciones cambian una vez que estás integrado en un grupo; es decir, que desde fuera las cosas no son lo que parecen. Quizá estos apuntes debemos tenerlos presentes a la hora de programar actividades para fomentar el asociacionismo, o deberían planteárselo incluso las asociaciones.

\section{RELACIÓN HOMBRES / MUJERES}

Vamos ahora a ver la relación que existe entre sexos, la relación de los hombres / mujeres según estén asociados o no asociados. 
Por los datos recabados de los que partimos en un principio, tenemos 102 asociados frente a los 494 que no lo están. Sin embargo comprobamos que a pesar de haber encuestado a más mujeres de forma totalmente aleatoria, como podemos verificar haciendo el recuento en el recuadro del principio referentes a los estudios, hay en proporción más hombres asociados, el 60 \% frente al 40\% que actualmente no están integradas en grupos. Hemos de reconocer que son datos contrarios a lo que en un principio podíamos esperar, ya que parece más propio que sean las mujeres las que más se asocien por el estereotipo de participativas y humanitarias. Sin embargo los datos nos han demostrado que, al menos en el campo estudiado, no se cumplen las expectativas.

\section{RELACIÓN EDADES ASOCIADOS}

Vemos en las próximas gráficas las relaciones existentes entre las edades de comienzo en las asociaciones dentro de la población que está asociada.

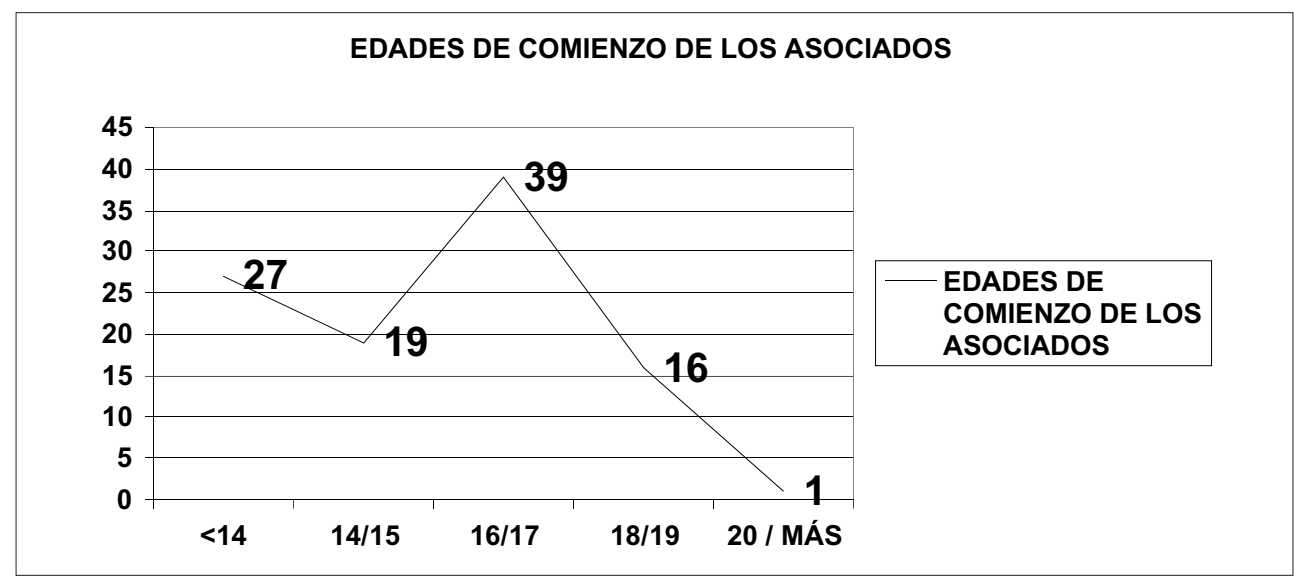

Vemos a partir de ahora en las próximas gráficas las relaciones entre las edades tanto de los asociados como de aquellos que no lo están. En ésta, vemos cómo la mayoría de los que actualmente continúan en un grupo juvenil se han animado entre los 16 y 17 años, aunque es significativo cómo también aumentaba el número de asociados antes de los 14 años, normalmente suele ser a los 12/13 por lo que nos han respondido. Suele coincidir con grupos parroquiales cuando comienzan las catequesis de confirmación. Aunque en un principio parezca que esto no significa nada, hemos de tener en cuenta que es uno de los momentos de reunión de grupos más o menos homogéneos que no pertenecen al mismo centro educativo, es decir que se crean nuevos y conocen nuevas personas. De aquí suelen salir pandillas de amigos que llevan la relación de grupo fuera de las paredes parroquiales.

También es significativa la caída que el asociacionismo sufre cuando se llega a plena adolescencia y juventud (18/19 años, y más marcada a los 20 o más años de edad). Si lo analizamos vemos que estas edades coinciden con los estudios universitarios en este caso, y por lo tanto las cuadrillas de amigos del instituto y parroquias 
tiende a separarse, cada uno se une a grupos diferentes y se centra en otros interesantes que normalmente van, en esta ocasión, en la línea de los estudios. Por lo tanto, el interés de permanecer en una asociación y el sacrificio que supone continuar con un grupo y unos intereses sociales que ahora se tambalean, comienza a hacer temblar los principios personales.

Todo ello demuestra que quizá de una forma inconsciente, la población siente una serie de necesidades que difieren en cierta medida de las que el resto de adultos , a pesar de organizar las actividades dedicadas a ellos. La capacidad de respuesta de una persona va a depender tanto de su capacidad personal según su desarrollo evolutivo como del conjunto de experiencias que conformen sus vivencias y desarrollo ambiental. Sin embargo todos confluyen en unas líneas evolutivas generales que coinciden a pesar del resto de discrepancias. Son estas líneas las que tenemos presentes en cualquier momento que contemos con organizar tareas para los jóvenes. Y esto es lo que ellos mismos nos están demandando a través de este estudio.

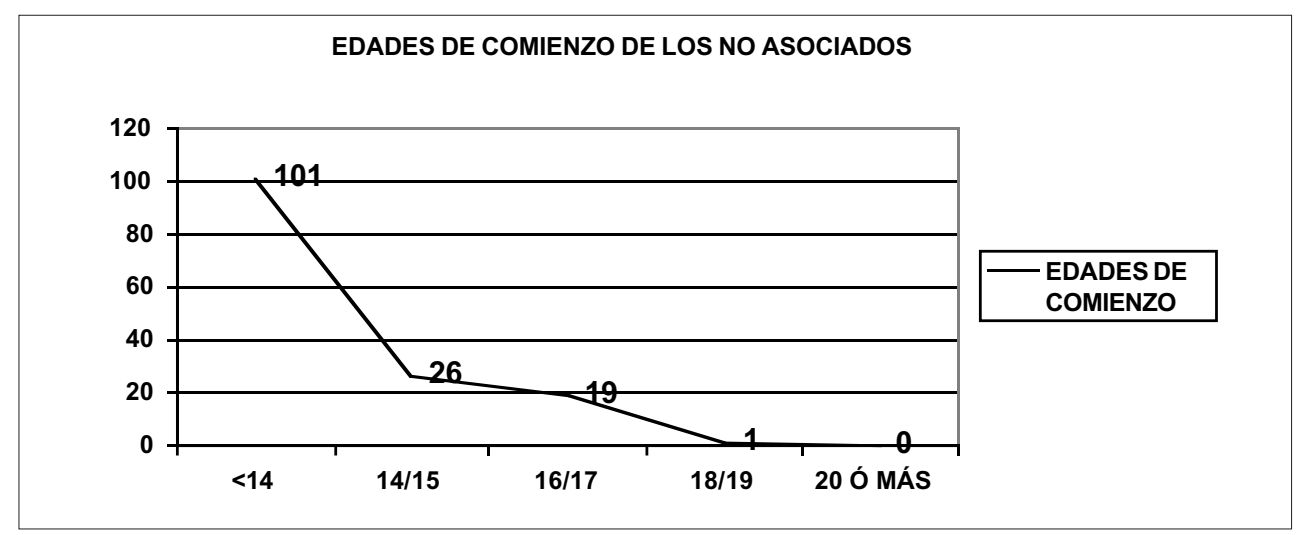

Sin embargo vemos ahora la gráfica de las edades de los no asociados pero que sí lo estuvieron alguna vez.

Si estudiamos ésta gráfica, vemos cómo los jóvenes se asociaron, de manera mayoritaria, antes de los 14 años, apunte que coincide con lo visto en la población opuesta; quizá los motivos sean los antes mencionados. A partir de ahí sufre un estrepitosa caída, para, al igual que con el otro grupo, continuar en niveles inferiores hacia los 18/19 años, y es este caso la nulidad para lo 20 o más.

Sabemos además por nuestros datos obtenidos en el estudio, las edades en las que los jóvenes dejaron de asistir a las asociaciones, que comprobamos que es en los 16/17 años, antes del comienzo de la universidad; datos que se relacionan directamente con los que ya hemos comentado inmediatamente antes (teniendo siempre en cuenta que las edades son bastante aproximadas, ya que no podemos afirmar con seguridad que sean las recogidas en los cuestionarios, dependiendo totalmente del recuerdo de los encuestados, por lo que tampoco las podemos tomar muy literales; pero son válidas en cuanto que nos dan una referencia bastante aproximada para nuestras conclusiones). 
Comprobamos pues que la relación de todas estas cifras da lugar a señalar que los jóvenes están interesados en asociarse antes de los 14 años, buscan una directriz que creen que pueden encontrarla en los grupos con otros semejantes. Seguramente aspectos relacionados directamente con las características de desarrollo evolutivo de la edad (pubertad / adolescencia). Sin embargo, por diversos motivos, posiblemente por los mismos que explicamos, o por los estudios, no encuentran lo que esperaban y deciden no continuar en las asociaciones. Y las abandonan antes de comenzar estudios universitarios.

\section{¿QUÉ TE GUSTA DE LAS ASOCIACIONES?}

Deberíamos centrarnos ahora por su interés sociológico, para el presente estudio y los posteriores, en los datos que nos aportan los jóvenes que afirman pertenecer a una asociación. Por ello presentamos un breve resumen de todos los datos recogidos y que presentan cierta relevancia.

De todos los asociados hemos sacado una relación de las distintas asociaciones a las que pertenecen para ver cuáles son los temas que más les preocupan y por lo que se mantienen agrupados. Recordamos que la mayoría de ellos son de grupos parroquiales, y les unen motivos religioso - sociales, es decir, permanecer en el grupo postconfirmación, o seguir con una cuadrilla que han conocido, y además motivaciones religiosas. Algunos son pertenecientes a inclinaciones políticas; y el resto se reparten entre scouts, Cruz Roja, y otras asociaciones e instituciones conocidas. También hay algunos que pertenecen a grupos culturales y asociaciones de alumnos del colegio en el que estudiaron o de la universidad.

Para encuadrar y realizar las correspondientes estadísticas, las hemos agrupado en cuatro grandes grupos: aquellas asociaciones que se dedican fundamentalmente a acciones humanitarias; otras que realizan actividades para niños y jóvenes, principalmente lúdicas y culturales; las que se centran en el cuidado de la naturaleza; y por último el apartado de otros, en el que tendrían cabida las religiosas y políticas.

La mayoría de ellas se centran en actividades para los jóvenes y los niños (así lo afirman 79 de los 102 asociados). Muy pocas son las que dedican entre sus actividades al cuidado y mantenimiento de la naturaleza. En segundo lugar queda el grupo de Otros (con el casi 44\%). Sin embargo, hay que tener en cuenta que para responder a esta pregunta tienen varias opciones, de manera que pueden marcar más de una solución. Por ejemplo, es común que los que pertenecen a grupos parroquiales hayan marcado Acciones humanitarias (el 38\%) y Actividades para niños y jóvenes, ya que entre sus actividades destacan esas. Otras instituciones, abarcan todas las posibilidades, por ello es que el número total de votaciones supera en número de asociados.

En cualquier caso, vemos que lo que más interesa son actividades de cualquier tipo que les llegue de cerca, en las que puedan sentirse integrados y realmente útiles y participativos.

No queda aquí los intereses que hemos intentado averiguar, vamos más hacia la profundidad de la investigación. Las actividades en las que nos detenemos no son específicas de un grupo, generalmente las instituciones están perfectamente organizadas y gestionadas de forma que abarcan todas las características y necesidades que 
consideran oportunas en factor de la población a la que va dirigida, siendo ante todo profesionales. Pero los jóvenes tienden a señalar aquellas que por diversos motivos resultan más llamativas para ellos por las circunstancias que viven o por el sentido y dirección que se le da a la propia actividad.

Quedan, por tanto actividades que no se hacen, o al menos no en la medida que se solicita. Esas serán las que vamos a detectar a continuación.

\section{¿QUÉ ACTIVIDADES TE GUSTARÍA HACER?}

Pero a pesar de haber visto cuáles son las actividades que les pueden interesar, sí que los jóvenes asociados aseguran que encuentran falta de otra serie de actividades que les gustaría hacer en más ocasiones, y que sin embargo no se hacen en la medida que ellos necesitan. Cabe ahora hacernos la siguiente pregunta, ¿escuchamos realmente lo que ellos necesitan? Y como consecuencia de ello, ¿puede ser por este motivo que desencadene en que no se asocien o dejen de asistir a los grupos juveniles? Quizá este estudio sirva para realizar el cambio que sea necesario para que realmente los jóvenes encuentren una línea en su vida, unos alicientes mayores que la bebida, el tabaco, las máquinas y otras malas compañías.

Tenemos en primer lugar que les gustaría realizar más charlas de temas interesantes para ellos, actuales, debates sobre la actualidad (al 28\%). Muchas veces no son temas interesantes aquellos que a nosotros sí nos parecen. Demandan más atención dirigida o centralizada en ellos como tema fundamental. Poco varían la decantación por las excursiones, convivencias y actividades de relaciones sociales, con un escasa diferencia ya que son el $27 \%$. Sí existe sin embargo, una notable variación, en cuanto a actividades solidarias se refiere, ayudar a los demás, ya que son sólo 19 de los 102 asociados de nuestro estudio. Por último, consideran otras actividades importantes que no suelen hacer, como deporte, teatro y otras actividades culturales (según opinan 15 asociados). Sin olvidar que entienden éstas programaciones en su forma pura, cuyo objetivo fundamental es el mismo de la realización.

Suelen preferir intercalar estas propuestas con las que ya realizan, no quieren dejar de hacer lo que actualmente hacen, pero sí simultanearlas con estas que aquí han expresado.

También es cierto, y hay que mencionarlo, que no han sido muchos los jóvenes que han respondido a esta pregunta, bien por despiste, bien porque están satisfechos con sus actividades actuales.

A esta pregunta ha respondido tan sólo la población asociada, es por ello que el número de respuestas resulte un poco menor de lo que en principio cabría esperar. La población contraria no puede opinar sobre unos aspectos que en principio no conocen lo suficiente.

A pesar de caer en la redundancia y casi la pesadez, se ha de insistir en la necesidad de relacionar datos, ya que hablamos de necesidades e intereses de personas, que ya por ese motivo es más difícil su cuantificación, y resulta más asequible la cualificación de las anotaciones pertinentes y comentarios que hemos concluido del estudio y que nos han aportado de forma totalmente participativa. 


\section{¿POR QUÉ TE HAS ASOCIADO?}

Entonces veamos cuáles son los motivos que han llevado a los jóvenes asociados a asociarse.

Tan sólo 4 de los encuestados que pertenecen actualmente a una asociación, continúa o se ha asociados por costumbre. Descartando el grupo de Otros, que luego comentaremos, el mayor número ya desmarcado del resto, el gran número de elecciones lo ha obtenido la labor social que de alguna manera desempeñan en la asociación, o que ellos lo tienen interiorizado como tal (tomando esta opción de respuesta casi el 50\%). Minoritarios en proporción, apenas el $21 \%$ de los encuestados lo hacen porque van sus amigos, y por permanecer juntos siguen en la asociación. Sin embargo, este dato no es incompatible con que además la asistencia se deba a otras causas, ya que la posibilidad de la multirespuesta hace más difícil concluir de forma unitaria. Habíamos dejado a un lado el apartado de Otros, con respuesta de 54 de los asociados (no olvidando la posibilidad de marcar más de una respuesta), porque una vez analizadas las conclusiones, en algunos casos detalladas que nos han proporcionado, vemos cómo muchos de ellos han respondido que lo hacen por diferentes motivos religiosos, sociales, etc. En definitiva, parece ser que a los jóvenes les inquietan más cosas de las que creíamos en un principio, tal como los estereotipos marcan. Ya es la segunda ocasión en que los estereotipos no se cumplen.

\section{Aportaciones Personales}

En este apartado pretendo hacer una pequeña conclusión personal sobre los resultados y comentarios que se han sucedido a lo largo de la investigación.

Como las hipótesis han reflejado, partíamos de la idea de que los jóvenes no están interesados en asociarse en algún grupo juvenil. Y como hemos comprobado esto es cierto. Sí ha habido un número representativo que afirma pertenecer a alguna entidad; sin embargo, en apartados anteriores hemos explicado que por los datos recogidos, la mayoría de esos grupos son parroquiales. Muchos de ellos nos mencionaron que pertenecen a grupos deportivos, pero lógicamente, éstos no pueden incluirse como asociaciones juveniles. Todo ello nos da una idea de que las cifras reales que barajamos son entonces menores.

Quisiera que los resultados obtenidos verdaderamente sirvieran para hacer todos una reflexión sobre el interés real de nuestro jóvenes en las instituciones; y ante eso qué hacer.

La elaboración y presentación de esta investigación ha sido una experiencia positiva que a la mayoría de los profesores con los que he tenido la posibilidad de tratar les ha parecido interesante e importante por descubrir la realidad juvenil. Pero también creo que queda mucho por hacer; un gran número de jóvenes no han podido ser encuestados por la limitación de nuestras previsiones; otros por no estar estudiando; y otros por no ser de Logroño. Es decir, que éste como tantos otros estudios necesita de una ampliación que permita que los nuevos datos que se retomen, confirmen los ahora expuestos y que además amplíe la población estudiada a diversos aspectos. Todavía queda mucho por hacer; ya hemos comenzado. 


\section{Referencias Bibliográficas}

CASERO ECHEVARRY, J.; (1994): "Guía práctica para la Organización y Gestión de Asociaciones". Valladolid. Secretariado de Publicaciones; Universidad de Valladolid.

EQUIPO CLAVE; (1994): “Aprendiendo a Organizar nuestra Asociación". Madrid. Ed. Popular.

INSTITUTO ANDALUZ DE LA MUJER; (1992): "Guía de asociaciones y Centros de Interés para las mujeres". Sevilla.

LOPEZ DE CEBALLOS, P. Y SALAS LARRAZÁBAL, M; (1988): "Formación de Animadores y Dinámicas de animación". Madrid. Ed. Popular.

LÓPEZ CEBALLOS, P; (1982): "Desarrollo participativo programado". Caracas (Venezuela). AVAD.

ORGANIZACIÓN Y GERENCIA ACTIVA; (1991): "Guía básica de las asociaciones"; OMEGA.

TSCHORNE, P, MAS, C Y REGOSO, J.L.; (1994): "Guía para la gestión de Asociaciones". Madrid. Ed. Popular.

Quiero expresar mi sincero agradecimiento y cordial saludo a D. Manuel Juarez Castelló, Dña. $M^{a}$ Jesús de Torre, y D. Juan $M^{\mathrm{a}}$ San Llorente, así como a todos aqueIlos que han hecho posible la realización material del presente estudio, que han colaborado de una manera totalmente desinteresada y altruista y que han prestado su tiempo y apoyo para que se pudiera llevar a cabo. 